Delft University of Technology

\title{
Adaptive State Estimation and Real-Time tracking of Aeroelastic Wings with Augmented Kalman filter and Kernelized Correlation Filter
}

Mkhoyan, T.; de Visser, C.C.; De Breuker, R.

DOI

10.2514/6.2021-0666

Publication date

2021

Document Version

Final published version

Published in

AIAA Scitech 2021 Forum

\section{Citation (APA)}

Mkhoyan, T., de Visser, C. C., \& De Breuker, R. (2021). Adaptive State Estimation and Real-Time tracking of Aeroelastic Wings with Augmented Kalman filter and Kernelized Correlation Filter. In AIAA Scitech 2021 Forum: 11-15 \& 19-21 January 2021, Virtual Event [AIAA 2021-0666] American Institute of Aeronautics and Astronautics Inc. (AIAA). https://doi.org/10.2514/6.2021-0666

Important note

To cite this publication, please use the final published version (if applicable).

Please check the document version above.

\footnotetext{
Copyright

Other than for strictly personal use, it is not permitted to download, forward or distribute the text or part of it, without the consent of the author(s) and/or copyright holder(s), unless the work is under an open content license such as Creative Commons.

Takedown policy

Please contact us and provide details if you believe this document breaches copyrights.

We will remove access to the work immediately and investigate your claim.
} 


\title{
Adaptive State Estimation and Real-Time Tracking of Aeroelastic Wings with Augmented Kalman Filter and Kernelized Correlation Filter
}

\author{
Tigran Mkhoyan*, Coen de Visser ${ }^{\dagger}$, Roeland De Breuker \\ Delft University of Technology, Delft, The Netherlands
}

\begin{abstract}
Advancements in aircraft controllers and the tendency towards increasingly lighter and more flexible aircraft designs create the need for adaptive and intelligent control systems. While lighter aircraft structures have the potential to show better structural and aerodynamic efficiency, they are also more susceptible to dynamic loads. A key aspect to account for the flexibility of the structure in a closed-loop design is aeroelastic state estimation and the feedback of wing motion (elastic states). A potential non-invasive approach to provide this measurement for control-feedback is visual tracking, with fuselage-mounted cameras observing the motion of the wing. In particular, high-speed visual tracking with correlation filters such as KCF (Kernelized Correlation Filter), allow to efficiently and robustly correlate between two samples with kernelized linear regression. A purely visual tracking filter however does not contain information regarding the dynamics of the system subject to tracking and may fail under marker loss and occlusion. To increase the robustness of the racking an EKF (extended Kalman filter) is added to the tracking filter acting as a KCF-EKF tracking couple. The Kalman filter is further augmented into augmented Kalman filter form, to allow joint on-line estimation of the model states and parameters. This proposed tracking approach is used to adaptively reconstruct the motion of a very flexible wing in real-time subject to gust excitation in the OJF (Open Jet Facility) wind tunnel at the Technical University of Delft. The method shows a good agreement with time and frequency domain analysis of the reference data measured by a laser vibrometer and demonstrated the effectiveness of KCF-AEKF couple under the presence of marker loss and model uncertainties for a model-free control approach.
\end{abstract}

\section{Introduction}

$\mathrm{T}$ He tendency towards increasingly flexible aircraft opens the possibility for better structural and aerodynamic efficiency, however with increasing flexibility and lightweight; also the susceptibility for higher dynamic loads is increased. Aeroservoelastic control design is one of the fields, where the flexibility of the structure and its interaction with aerodynamic loads is closely coupled and challenging. In such controller designs, monitoring the entire wingspan can be crucial for proper delegation of control actions. This may involve installing many conventional accelerometers that are likely subject to noise and bias, suffer from lack of space, must deal with certification requirements and face challenges associated with correct geometric placement. A smart sensing approach is desired for those examples of wing structures that rely on novel types of sensors to provide feedback to an intelligent controller.

A solution that can greatly reduce the complexity associated with hardware installation, and provide the flexibility needed for employing novel state estimation methods, is aeroelastic state estimation by visual methods. An illustration of aeroelastic state estimation using visual data is shown in Fig. 1. The use of visual information for observing deformations has been successfully implemented on wind tunnel models in early studies [1], and has also seen wide application in robot manipulation [2]. However, in recent years, the capability in terms of on-board computation and the camera quality has immensely increased, while the hardware has become more compact $[3,4]$. This opens the door for numerous embedded applications using a camera as a sensor. In particular, fuselage-mounted camera systems can provide significant advantages for flexible aircraft systems, save costs associated with installation, certification,

\footnotetext{
*Ph.D. student, Faculty of Aerospace Engineering, Aerospace Structures and Materials department, T.Mkhoyan@tudelft.nl, P.O. Box 5058, 2600GB Delft, The Netherlands.

${ }^{\dagger}$ Assistant Professor, Faculty of Aerospace Engineering, Control and Operations department, C.C.deVisser@ tudelft.nl, P.O. Box 5058, 2600GB Delft, The Netherlands

Associate Professor, Faculty of Aerospace Engineering, Aerospace Structures \& Computational Mechanics, R.DeBreuker@tudelft.nl, P.O. Box 5058, 2600GB Delft, The Netherlands
} 


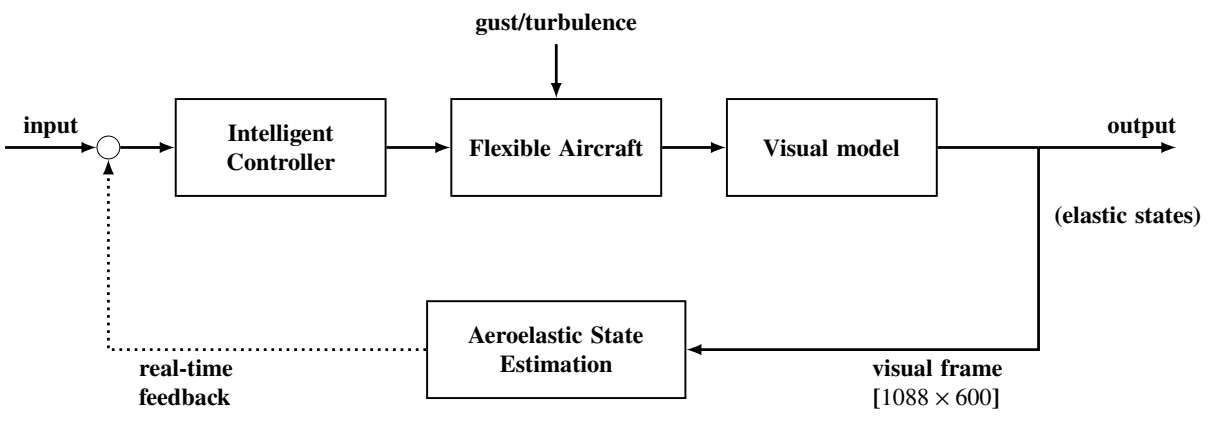

Figure 1 State estimation setup with visual tracking in a controller scheme.

and have the potential of being non-invasive and universally applicable. This type of smart sensing system is also of particular importance for over-actuated and over-sensored morphing designs such as the SmartX [5]. Furthermore, image data is a rich source of information; collected over a period of time, it unlocks the opportunity to approach the state estimation from a new perspective using machine learning methods. While many suitable tracking methods exist for marker detection, purely visual tracking methods have no knowledge of the underlying dynamics of the system. The disadvantage is that a visual filter alone may fail in the presence of occlusions in the image, show sensitivity to lighting conditions and produce high errors under large deformations of the structure. To correctly estimate the motion of the structure, an adaptive approach is required that is aware of the system exhibiting dynamic behaviour and attempts to estimate system parameters. To account for this shortcoming, this study describes a tracking method, consisting of a purely visual filter, a high-speed Kernelized Correlation Filter (KCF), with an Augmented Extended Kalman Filter (AEKF). The combined tracking KCF-AEKF filter, adaptive estimates on-line both the state and the parameters of the system subject to disturbance, exhibiting oscillatory motion. The addition of the AEKF and parameter estimation allows to reduce the dependency of the model, making this approach very suitable for closed-loop control of uncertain systems, and very flexible systems exhibiting non-linear response. The method is validated experimentally on a real-time image stream of a very flexible wing subjected to gust excitation in the OJF (Open Jet Facility) at the Delft University of Technology.

\section{Methodology}

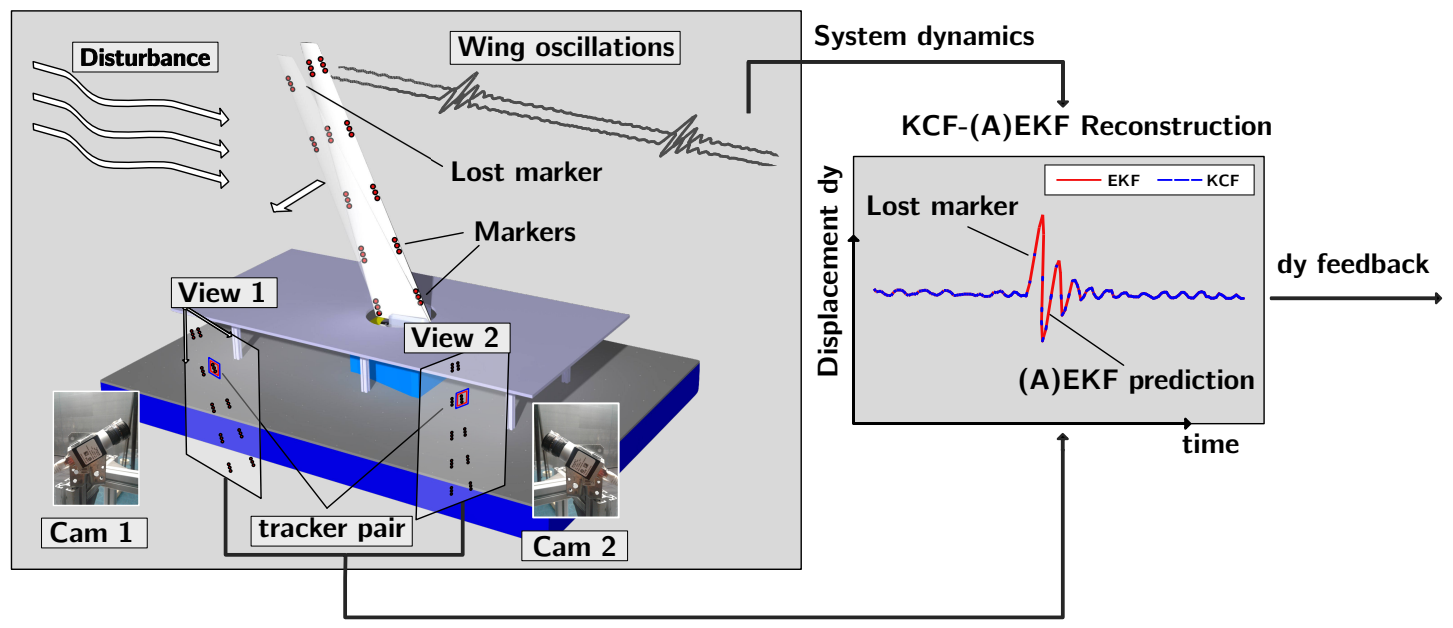

3D Reconstruction

Figure 2 Illustration of the wing tracking and reconstruction approach using KCF-AEKF. 
The method is composed of a visual tracking method, combined with adaptive state estimation. The visual tracking uses state-of-the-art high-speed KCF (Kernelized Correlation Filter). The tracker is augmented with AEKF (Augmented Extended Kalman Filter), allowing on-line estimation of with augmented time-varying mass, stiffness, and damping states. Furthermore, the state and state derivative feedback signals are provided by the visual system in real-time.

\section{A. KCF: Visual Filtering}

KCF belongs to a family of correlation filter where the objective is to find the correlation between two samples. When this is applied to a tracking problem, the correlation filter observes the correlation of the original target object defined in a patch moving trough future sample images. Regular correlation filters do not allow for updating of the initial target object, KCF, in contrast, updates the model of the object on-line using a kernelized linear regression, thereby retaining robustness against object mutation (change of appearance through samples). Another property of the $\mathrm{KCF}$ is the utilisation of the properties of circulant matrices and kernel functions reducing the computation to an order $O(\log (n))$ versus $O\left(n^{3}\right)$ for typical correlation filters. This allows the KCF tracker to reach high speeds and reduce the memory storage for the computation. Below a summary is given of the KCF methodology [6].

\section{Summary of the KCF methodology}

The aim in the tracking problem is to learn to predict the mutation, presence or absence of the target object in the future frames. The learning method in $\mathrm{KCF}$ is based on the linear ridge regression [6]:

$$
\min _{w} \sum_{i=1}^{n}\left(f\left(\boldsymbol{x}_{i}\right)-y_{i}\right)^{2}+\lambda\|\boldsymbol{w}\|^{2}
$$

Where, $x_{i}$ are the samples, $y_{i}$ are the regression targets, $w$ is a vector of weights, $\lambda$ the regularization parameter to prevent overfitting and the objective is to find a function $f((z))$ that minimizes the squared error. The latter function typically adapts the following model:

$$
f(z)=(w)^{T} z
$$

The minimisation can be formulated in the closed-form which can be represented as a convex function with a unique solution as:

$$
\boldsymbol{w}=\left(X^{T} X+\lambda I\right)^{-1} X^{T} \boldsymbol{y}
$$

Here the matrix $X$ contains the vector of sample images $x_{i}$ per row, $\boldsymbol{y}$ contains the regression targets $y_{i}$ as elements, and $I$ is the identity matrix. The above formulation involves solving a large system of linear equations, which can work for a problem for real-time tracking. To elevate this KCF uses circulant matrices composed of shifted samples of $\boldsymbol{x}$ of the object of interest and performs the computation of all possible cyclic shifts efficiently in the Fourier domain. To transition into the Fourier domain, the term $X^{T}$ is replaced by the Hermitian transpose $X^{H}=(X *)^{T}$ and $X *$ represents the complex conjugate of $X$. The pattern by which the circulant matrices is generated is deterministic and fully determined by the generating vector $x$. A powerful property of the circulant matrices arising from the way it is structured is that they become diagonal in the Fourier domain. Denoting the Discrete Fourier Transform (DFT) as $\hat{\boldsymbol{x}}=\mathcal{F}(\boldsymbol{x})$, and matrix $F$ as the constant DFT matrix not depending on input vector, the resulting matrix $X$ in Fourier domain becomes [7]:

$$
X=F \operatorname{diag}(\hat{\boldsymbol{x}}) F^{H}
$$

This formulation allows the computational convenience of element-wise operation as the matrices are diagonal. Making further use of the symmetric properties of the circulant matrices and defining a dot-wise product operator also the term $X^{H} X$, and $\mathrm{u}$ can be represented in compact form as:

$$
X^{H} X=F \operatorname{diag}\left(\hat{\boldsymbol{x}}^{*} \odot \hat{\boldsymbol{x}}\right) F^{H}
$$

Here the dot product between the brackets represents the autocorrelation of input $\boldsymbol{x}$ in Fourier domain. Typically autocorrelation of a time signal represents the shifted time lags with itself, applied to image data; this represents the spacial 'lag'. Combining all of the above, the minimisation problem can be formulated as: 


$$
\hat{w}=\frac{\hat{a}^{*} \odot \hat{z}}{\hat{a}^{*} \odot \hat{a}+\lambda}
$$

A final step in the methodology of the KCF is the introduction of kernel functions in the linear ridge regression by applying the so-called 'kernel trick'. In many application, data is much easier to separate in higher-dimensional space. The kernel trick allows to efficiently evaluate the dot product in higher dimensional space without the need for costly transformations of the input vectors. Following the methodology described in [6], the kernelized formulation for coefficient updates gives:

$$
\hat{\boldsymbol{\alpha}}=\frac{\hat{\boldsymbol{y}}}{\boldsymbol{k}^{x \hat{x}}+\lambda}
$$

Here the hat terms similarly represent the vectors in the Fourier domain, $\boldsymbol{k}^{x x}$ represents the first row in the kernel matrix defined as $K=C\left(k^{x x}\right)$ and $\hat{\boldsymbol{\alpha}}$ represents the vector of the coefficients $\alpha_{i}$ in this higher dimensional space, similar to the formulation of weights in eq. 3 :

$$
\boldsymbol{\alpha}=(K+\lambda I)^{-1} \boldsymbol{y}
$$

To allow detection of the target object, the regression function $f(z)$ needs to be evaluated at several image locations or candidate patches. The candidate patches can be constructed by adopting the cyclic shift model, allowing to define the kernel matrix as $K^{z}=C\left(\boldsymbol{k}^{k z}\right)$. Here, $\boldsymbol{x}$ is the base sample, $z$ the base patch and $k^{x z}$ is the kernel correlation of the two vectors, or the relative shift of two vectors. Evaluating the regression function using circular shifts is effectively a spacial filtering operation. This operation can be most efficiently performed in fourier domain. The regression function is then represented as a linear combination of kernel values $k^{x z}$ and learned coefficients $\alpha$ :

$$
\hat{f}(z)=\hat{k}^{x z} \odot \hat{\alpha}
$$

Various kernel functions are possible. To relax the computational effort KCF proposes an Radial Basis Function (RBF) gaussian kernel function $k\left(x, x^{\prime}\right)=\exp \left(-\frac{1}{\sigma^{2}|| x-x^{\prime} \|^{2}}\right)$ arriving to the following kernel correlation form and $O(n \log n)$ computational time:

$$
\boldsymbol{k}^{x x^{\prime}}=\exp \left(-\frac{1}{\sigma^{2}\|x\|^{2}+\left\|x^{\prime}\right\|^{2}-2 \mathcal{F}^{-} 1\left(\hat{x}^{*} \odot \hat{x}^{\prime}\right)}\right.
$$

For each future frame the coefficients learned in the previous frame can be updates by linear integration with new parameters. This allows to build a memory into the model that is controllable by the regularization parameter $\lambda \in[0,1]$ :

$$
\hat{\boldsymbol{\alpha}}(t)=\lambda \hat{\boldsymbol{\alpha}}+(1-\lambda) \hat{\boldsymbol{\alpha}}(t-1)
$$

\section{B. EKF and AEKF: state and parameter estimation}

\section{Simplified Dynamic model}

The simplest model of a Kalman filter which can be used in unison with visual racking has linear motion dynamics. Since the wing typically exhibits oscillatory motion under aerodynamic input loads, the performance and the robustness of the visual tracking can be improved by extending the simplified dynamics of a moving linear particle to a typical spring-mass damper system. This robustness is in particular of importance when the LEDs fail for longer period of time (longer than a half a period of one typical oscillation) and momentarily no observation is available of the visual markers. The clamped wing, structurally representing a clamped beam model beam, will oscillate around its equilibrium, under external excitation. If the intervals of led loss are small, the moving particle is robust enough to interpolate the motion, however, it will not be able to capture the oscillations dynamics. This oscillatory dynamics can be captured by a relatively simple linear 1-DOF 2 nd order mass damper system, where we look at displacement $y(t)$ of a point mass. The general differential equation is given as:

$$
\ddot{y}(t)=-\frac{c}{m} \dot{y}(t)-\frac{k}{m} y(t)
$$


In the state space form we have the following:

$$
\frac{d}{d t}\left[\begin{array}{l}
y_{k} \\
\dot{y}_{k}
\end{array}\right]=\left[\begin{array}{cc}
0 & 1 \\
-k / m & -c / m
\end{array}\right]\left[\begin{array}{l}
y_{k} \\
\dot{y}_{k}
\end{array}\right]
$$

It must be noted that in this form we consider the homogeneous form for two reasons: (i) gust (external) input cannot be measured directly and (ii) the aim is to maintain the Kalman filter as model-free as possible. The gust input can be modelled as initial displacement in this Kalman filter form.

\section{Discrete KF formulation}

To deal with time intervals of image capture, $h=\Delta t$, a discrete Kalman filter form must be formulated. Using a typical Euler integration given in this from of the state $x$ :

$$
\frac{d(x(t)}{d t}=\dot{x}(t)=f^{\prime}(x(t))=\frac{x(t+h)-x(t)}{h}
$$

Rewriting gives the definition of next time step:

$$
x(t+h)=x(t)+f^{\prime}(x(t)) h
$$

Now in discrete form using $k=t+h$ :

$$
x_{k}=x_{k-1}+\dot{x}_{k-1} h
$$

The Kalman Filter contains the states $\bar{x}_{k}=\left[\begin{array}{ll}y_{k} & \dot{y}_{k}\end{array}\right]^{T}$. Each state of the Kalman Filter can be rewritten in a discrete recursive form. The states of the Kalman filter are:

$$
\begin{aligned}
& y_{k}=y_{k-1}+\dot{x}_{k-1} h \\
& \dot{y}_{k}=\dot{y}_{k-1}+\ddot{y}_{k-1} h
\end{aligned}
$$

Using expression for the acceleration, the velocity can be rewritten into:

$$
\dot{y}_{k}=-k / m \cdot y_{k-1}-(1-c / m \cdot h) \cdot \dot{y}_{k-1}
$$

Now the following system state matrix is obtained:

$$
\bar{x}_{k}=\left[\begin{array}{c}
y_{k} \\
\dot{y}_{k}
\end{array}\right]=\left[\begin{array}{cc}
1 & h \\
-k / m & -(1-c / m \cdot h)
\end{array}\right]
$$

Where the output is:

$$
\bar{z}_{k}=\left[\begin{array}{ll}
1 & 0
\end{array}\right] \bar{x}_{k}
$$

While changing the model to this oscillator gives more robustness against LED marker loss, it introduces other complications:

- The model has now become slightly more complex and model parameters $m, c, k$ need to be estimated.

- Additionally, we can no longer work with arbitrary time step, and the actual time step in between the image frames is required. This time step difference must set the discrete sampling, $d t$ of the Kalman Filter at each iteration of the tracker algorithm.

- Furthermore, transformation is now required to go from $x, y$ frame coordinates to the frame of reference for the modelled oscillator system.

However, the above mentioned points implicitly also imply that the the more complex model approach, may no longer be model free. To overcome this, the proposed approach is to make the model non-linear (time varying in $c, k$ and $m$ parameters) and use AEKF to estimate these model parameters. The way this can be achieved, is to augment the previous state vector $x_{k_{s y s t e m}}$ with unknown model states $p_{k_{\text {model }}}$. For full time-varying spring-mass-damper system this will become, $\bar{x}_{k}=\left[\begin{array}{lll}\overline{x_{k s y s t e m}} & \overline{p_{k \text { model }}}\end{array}\right]=\left[\begin{array}{lllll}y_{k} & \dot{y}_{k} & K_{k} & c_{k} & m_{k}\end{array}\right]^{T}$. 


\section{AEKF formulation}

To rewrite the model into an augmented form and perform parameter estimation, the system parameters can be made a function of time. The differential equation can take the following form:

$$
\ddot{y}(t)=-\frac{c(t)}{m(t)} \dot{y}(t)-\frac{k(t)}{m(t)} y(t)
$$

One commonly used assumption for the augmented model is to assume that the derivative of model parameters is zero: $\dot{p}_{k}=\left[\begin{array}{lll}0 & 0 & 0\end{array}\right]^{T}$.

Now the following non=linear system matrix is obtained:

$$
\bar{x}_{k}=\left[\begin{array}{c}
y_{k} \\
\dot{y}_{k} \\
K_{k} \\
c_{k} \\
m_{k}
\end{array}\right]=\left[\begin{array}{c}
y_{k-1}+\dot{x}_{k-1} h \\
-K_{k-1} / m_{k-1} \cdot y_{k-1}-\left(1-c_{k-1} / m_{k-1} h\right) \cdot \dot{y}_{k-1} \\
K_{k-1}+0 \cdot h \\
c_{k-1}+0 \cdot h \\
m_{k-1}+0 \cdot h
\end{array}\right]
$$

In order to solve AEKF model at each time step, the Jacobian of the system matrix w.r.t time dependent states must be constructed. This allows to linearise the model at each time step as follows:

$$
J\left(\bar{x}_{k}\right)=\begin{array}{ccccc}
1 & h & 0 & 0 & \\
-K_{k-1} \cdot m_{k-1}^{-1} \cdot h & 1-c_{k-1} \cdot m_{k-1}^{-1} \cdot h & m_{k-1}^{-1} \cdot y_{k-1} \cdot h & -m_{k-1}^{-1} \cdot \dot{y}_{k-1} \cdot h & m_{k-1}^{-2} \cdot c_{k-1} \cdot \dot{y}_{k-1} \cdot h-m_{k-1}^{-2} \cdot K_{k-1} \cdot y_{k-1} \\
0 & 0 & 0 & 0 & 0 \\
0 & 0 & 0 & 0 & 0 \\
0 & 0 & 0 & 1
\end{array}
$$

The output of interest remains the measured the displacement, however, now the system parameters are also estimated at each time step as output.

\section{Image processing and reconstruction pipeline}

\section{Clustering}

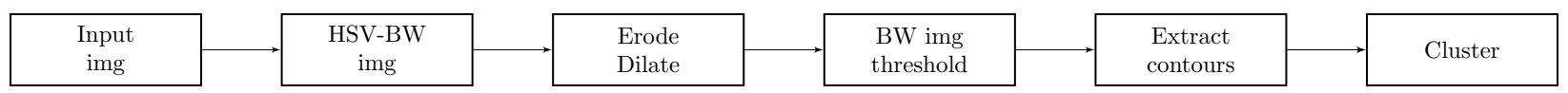

Figure 3 Schematic of HSV filtering, such that a BW image is obtained for subsequent clustering.

To track visual markers, a processing pipeline is required. The initial step is to correctly detect and cluster the markers. For this purpose, a machine learning approach is developed. The method utilizes DBSCAN and inverse formulation of DBSCAN proposed in the earlier study [8]. DBSCAN was found to be particularly suitable for the task due to its unsupervised nature, namely (i) minimum needed domain knowledge, (ii) ability to find clusters of varying size and (iii) ability to deal with noise. The image filtering pipeline for the clustering process is shown in Fig. 3 . Unsupervised clustering method was essential due to the periodic failure of the led markers installed on the wing. This is illustrated in Fig. 4. 

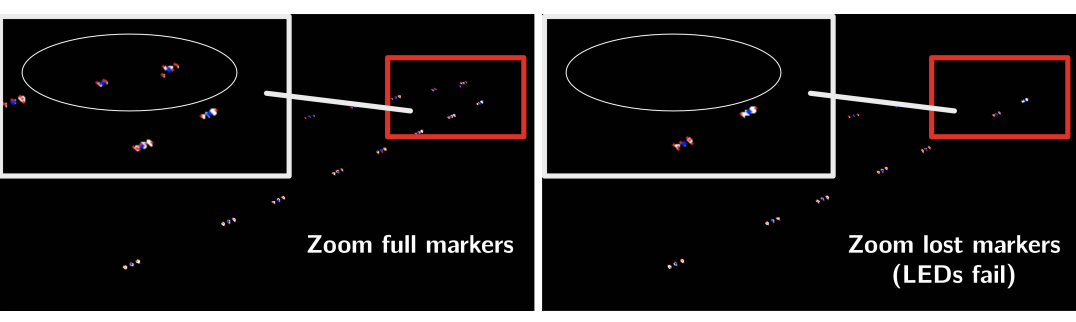

(a) Clustering of a complete set of markers.

(b) Clustering of an incomplete marker set (LEDs are lost), but unsupervised clustering is capable of finding the correct number of clusters.

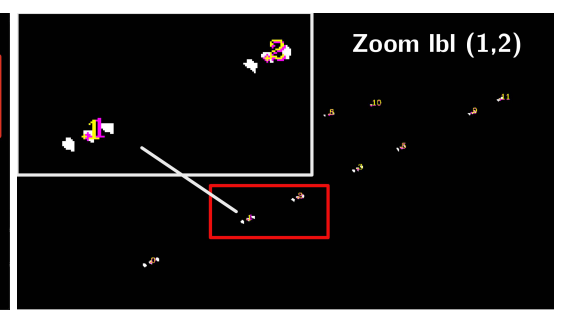
ple), and the Disjoint-set data structure (yellow); the zoomed region shows marker labels 1, 2 (lbl (1,2)).

Figure 4 Difference in mask obtained after thresholding of an incomplete (left) versus full (middle) set of markers (due to periodic failure of LEDs), showing the necessity of unsupervised clustering. The red dots are contours of the mask and blue is the centroid. The image on the right shows the result of clustering.

\section{Radial sorting}

To obtain a geometrical representation behind the detected clusters, a radial sorting algorithm is proposed in the processing routine after clustering operations. The algorithm is initiated by finding the centroid $\overline{\mathbf{c}}_{c p}$ of the cluster centres (a cloud of points) $\mathbf{P}(x, y)$, then obtaining a radially sorted distribution, a so-called convex radial hull, $\mathbf{P}_{\theta \text { hull }}$, of $n$ indices, such that the outline of the hull has continuous connectivity.

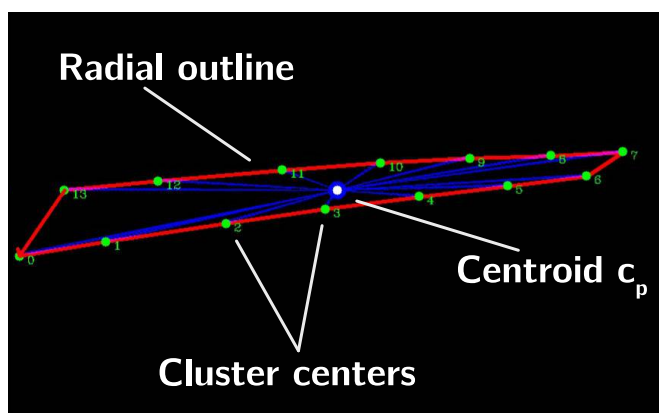

Figure 5 Radial sorting algorithm process. The cluster centres are green dots, the convex radial hull is the red outline connecting the centroid.

\section{Reconstruction}

A high-level overview of the full tracking pipeline is shown in Fig. 6.

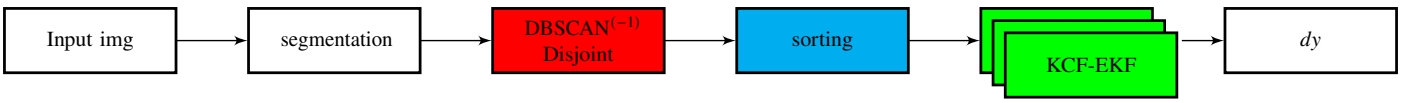

Figure 6 Overview of the full tracking pipeline.

The red block is the clustering algorithm (DBSCAN/DBSCAN ${ }^{-1} /$ Disjoint) implemented in this study. The green blocks represent an independent tracking filter and Kalman filter duos (KCF-AEKF), that run in parallel to keep track of markers through a sequence of images. The output is the displacement of the marker in $x, y$ pixel coordinates of the frame. The reconstruction is the final step that relates the displacements of corresponding markers in two frames and reconstructs the 3D displacement. The reconstruction process can be inferred from the schematic of the camera setup shown in Fig. 7. Further details regarding the 3D reconstruction can be found in a previous study by Mkhoyan et al. [9] and [10]. 


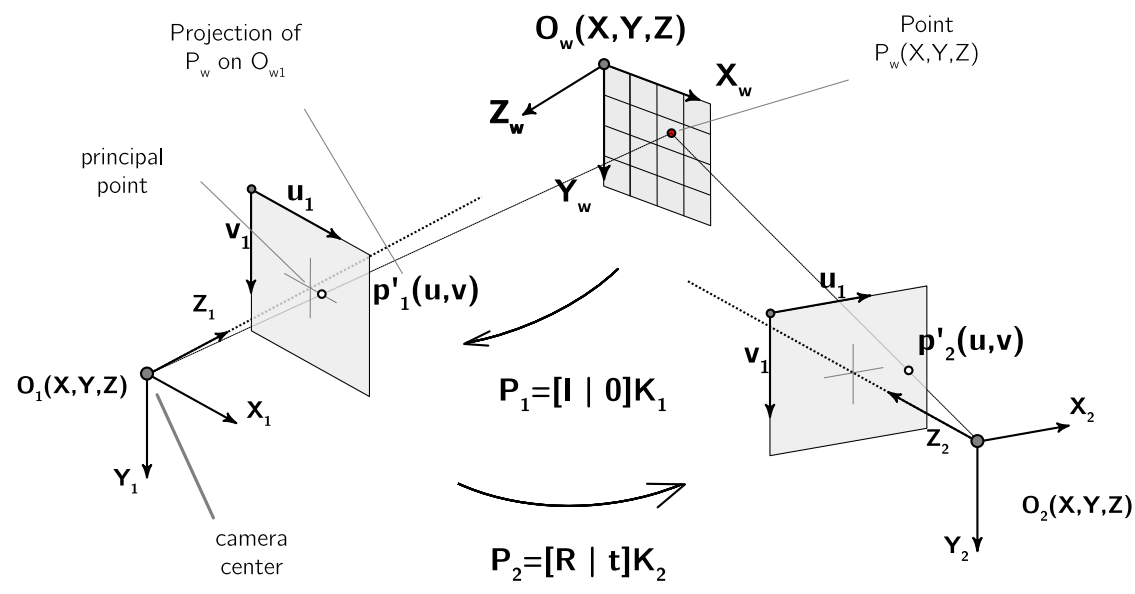

Figure 7 Schematic of the stereo camera setup and the coordinate systems for 3D reconstruction.

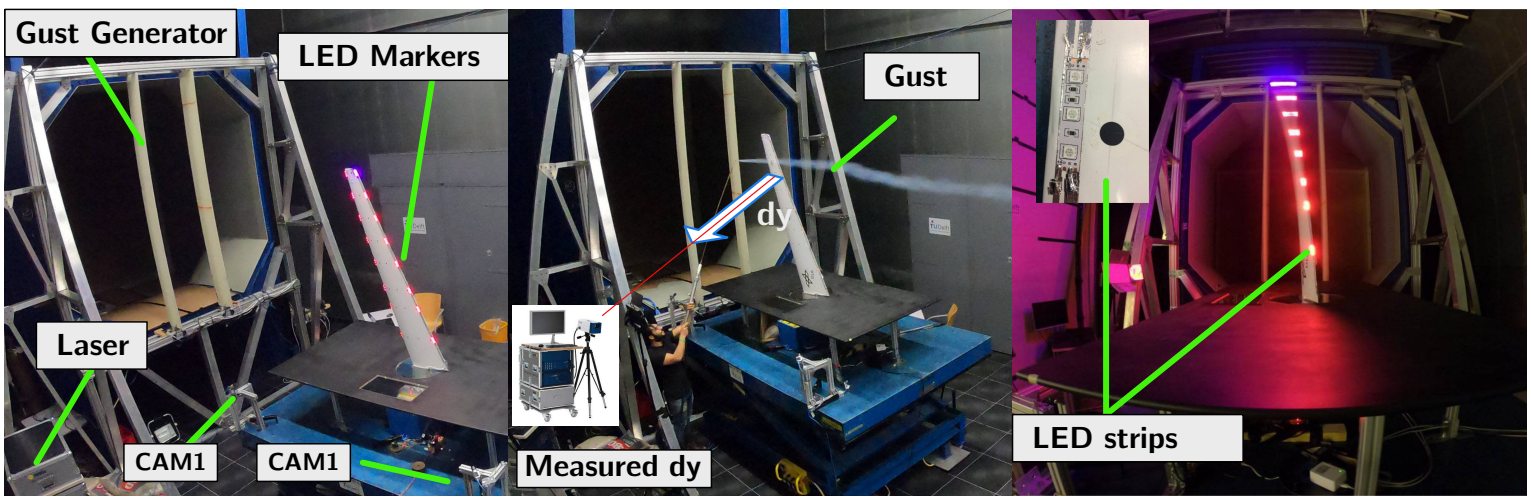

Figure 8 The Open Jet Facility (OJF) [11] with the gust generator mounted in front of the test section. Wing flexing with LED strips installed on the right.

\section{Experimental Setup}

To collect the experimental data and test the proposed methods, a flexible wing was equipped with an array of visible (active) LED markers and subjected to gust excitation. The study is the continuation of a previous study [8] on smart sensing methods for control of flexible aircraft.

\section{A. Apparatus}

The experiment was conducted in the Open Jet Facility (OJF) located at the Aerospace Engineering faculty of the Delft University of Technology [11]. The Open Jet Facility (OJF), is a closed-circuit low-speed wind tunnel, with an octagonal test section of $285 \times 285 \mathrm{~cm}^{2}$. The wind-tunnel facility shown in Fig. 8, was equipped with a gust generator [12] composed of two gust vanes actuated in parallel. The gust vanes allowed generating discrete (1-cosine), harmonic and sweep signals, resulting in wing gusts on top of nominal wind tunnel free stream velocity. The gust vanes were operated at maximum of $5-7 \mathrm{~Hz}$ for gust vane angle of $\alpha_{g}<= \pm 15^{\circ}$, and $10-15 \mathrm{~Hz}$ for vane angles $\alpha_{g}<= \pm 10^{\circ}$. The freestream velocity in the wind tunnel was maintained by a $500 \mathrm{KW}$ electric engine. The maximum flow velocity available in the wind tunnel is $35 \mathrm{~m} / \mathrm{s}$; however, the theoretical performance limit is around $30 \mathrm{~m} / \mathrm{s}$.

To measure the deflections resulting from the gust excitation a Polytech PSV-500 laser vibrometer system was used with a resolution (RMS) of $200 \mu \mathrm{m} / \mathrm{s}$ [13]. In total 8 markers were measured by the system as shown in figure 9a. 


\section{B. Wing model}

The wing used in the experiment was a forward-swept tapered wing, built of glass fibre reinforced epoxy material, referred to as the Allegra wing. The design of the wing allows for large tip displacements, up to $20 \%$ for $10^{\circ}$ of Angle of Attack (AoA) and $50 \mathrm{~m} / \mathrm{s}$ flow velocity [14]. The wing was clamped on one-side on a sturdy table under a fixed angle of attack of $4^{\circ}$. Detailed information about the wing can be found in [15].

The wing was equipped with 16 LED markers. Each LED marker consisted of 3 sub-LED units, providing three distinct bright light sources per marker. In the experiment, a 1-cos gust signal and a frequency sweep signal were used.

\section{Experimental conditions}

For all the experimental conditions cases, the experimental setup was kept constant. The wind tunnel test consisted of two experimental conditions, (i) static and (ii) dynamic, two camera conditions (perfect, dark) and two wing configurations (no mass, mass). The latter two wing configurations were introduced to investigate the effect of wing mass matrix and wing inertia change to tracking performance and the parameter estimation of the mass parametters in the AEKF oscillator model. The purpose of the camera conditions was to assess the robustness of visual tracking in the presence of external visual disturbances. The camera conditions were designed to simulate realistic lighting conditions such that the feasibility of the method in-flight could be evaluated. For all three conditions, the illuminance of the room was to be measured to establish a baseline sensitivity for light intensity and tracking performance. An overview of independent and dependent variables for the wing configuration and camera is given in table 1.

Table 1 Parameters of the wing and camera setup in the wind tunnel experiment.

\begin{tabular}{lll|ll} 
& IV's (controlled) & & DV's (measured) & \\
\hline & parameter & unit & parameter & unit \\
\hline \multirow{2}{*}{ Camera } & $I_{\text {led }}$ (led intensity) & $\mathrm{W}$ & $I_{\text {room }}$ (illuminance room) & lux \\
& $N_{\text {trackers }}$ & - & $H$ (camera frame) & pixel \\
\hline \multirow{4}{*}{ Wing } & $\alpha$ AoA & $\mathrm{deg}$ & $d_{x}$ (displacement-x) & $m$ \\
& $v_{o}$ (flow velocity) & $m / s$ & $d_{y}$ (displacement-y) & $m$ \\
& $m$ (wing tip mass) & $\mathrm{kg}$ & error displacement & $m$ \\
& $f_{g}$ (gust frequency.) & $\mathrm{Hz}$ & & \\
& $d_{g}$ (gust vane angle.) & $\mathrm{deg}$ & & \\
\hline
\end{tabular}

\section{Camera conditions}

Two visibility conditions were used, $\mathrm{C} 1$ dark conditions (night visibility, low background light corresponding) and C2 bright condition (daylight visibility, high background lighting). The visibility was selected to study the effect of tracking and sensitivity of HSV filtering.

\section{Motion conditions}

The motion conditions composed of two cases, static and dynamic. In both cases, each condition was repeated for different camera configurations. The dynamic motion conditions were selected such that it produced a high dynamic response from the wing and thus more pixel activity in the image. The gust vane frequency of $5 \mathrm{~Hz}$ was close to the wing's natural frequency at the given mass configuration.

The objective of the static case was to assess the ability to (i) extract absolute displacements and (ii) monitor any steady-state error (bias) developed during the measurement runs in tracking the position of the markers.

In the dynamic case, the aim was to assess the (i) robustness of the tracking and (ii) monitor the measurement bias (drift) developed over time. The robustness indicator is the ability of the tracker to maintain the tracked marker for high-frequency oscillations. The aim was to cover the aerodynamic behaviour of the wing for typical free stream velocities and angles of attack. The test matrix of the experimental motion conditions is given in table 2 . 
Table 2 Test matrix of the experimental motion conditions.

\begin{tabular}{|c|c|c|c|c|c|c|}
\hline condition & signal & $V_{0}[\mathrm{~m} / \mathrm{s}]$ & AoA [deg] & Gust freq. $[\mathrm{Hz}]$ & Gust vane a. $[\mathrm{deg}]$ & Camera c. \\
\hline static & & {$[5,10,20,25,30]$} & {$[2]$} & - & - & {$[\mathrm{C} 1, \mathrm{C} 2]$} \\
\hline dynamic (gust) & & {$[10,30]$} & {$[2]$} & {$[1,2,3,4,5,5.6]$} & {$[5,6,7,8,9,10]$} & {$[\mathrm{C} 1, \mathrm{C} 2]$} \\
\hline dynamic (sweep) & & {$[10,30]$} & {$[2]$} & $\infty$ & {$[5,10]$} & {$[\mathrm{C} 1, \mathrm{C} 2]$} \\
\hline
\end{tabular}

\section{Visual tracking setup}

An overview of the hardware used for dataset collection is shown in Fig. 9b. The dataset was recorded with two GigE acA1300-75gc Basler ethernet cameras with 1300 CMOS 1.3 megapixel $(280 \times 1024$ pixels) sensor [16]. The cameras were equipped with Computar $12 \mathrm{~mm}$ F1.4 2/3" P IRIS lenses [17] and were positioned in a stereo setup to observe the markers from two viewpoints. The resulting image was cropped to $1088 \times 600$ pixels and streamed in 3 channel RGB format synchronously via real-time PTP triggering protocol over the ethernet. A Power over Ethernet (PoE) smart switch GS110TP from NETGEAR, provided both the power, $3.5 \mathrm{~W}$ (per camera unit), as well as the GigE capability to stream the images up to 140 Frames Per Second (FPS).

The processing power and image capture were delivered by an embedded computing system from NVIDIA, the Jetson TX2, equipped with NVIDIA Pascal architecture with 256 NVIDIA CUDA cores and 1.3 TFLOPS (FP16), Dual-core Denver 2 64-bit CPU and quad-core ARM A57 complex [18]. The Jetson TX2 is designed for embedded applications using Artificial Intelligence (AI) and Computer Vision (CV) and operates on Ubuntu 16.04 LTS allowing flexibility in code deployment. The application developed for this study was programmed in $\mathrm{C}++$ and deployed on the device. For the development the Basler C++ Pylon API [16] and OpenCV open-source computer vision library [19]. The image and tracking data were extracted and plotted in using the OpenCV-Matlab parsing interface tmkhoyan/cvyamlParser [15].

Code development, testing and assessment was done using standard Dell Optiplex 7400 and 2.3 GHz Intel Core i5 $16 \mathrm{G}$ MacBook and the Jetson TX2. A part of the code, dataset and tools developed in the scope of the study are available under the repositories tmkhoyan/adaptiveClusteringTracker [8, 10] and tmkhoyan/parallelTrackingTBB [9, 20].

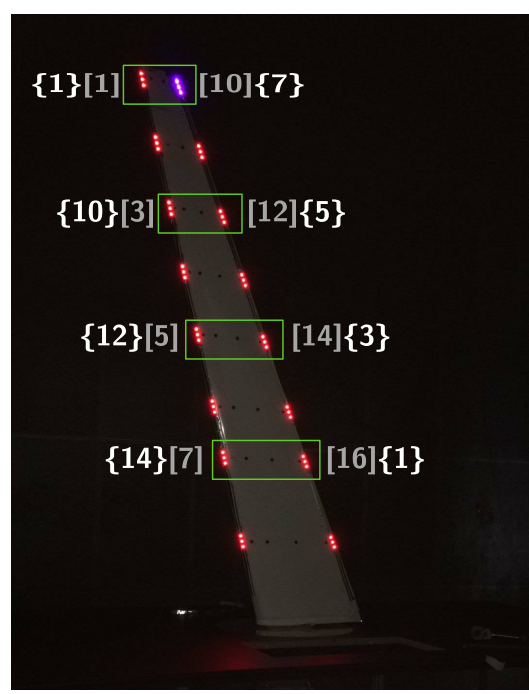

(a) Active marker locations (in green square braces).

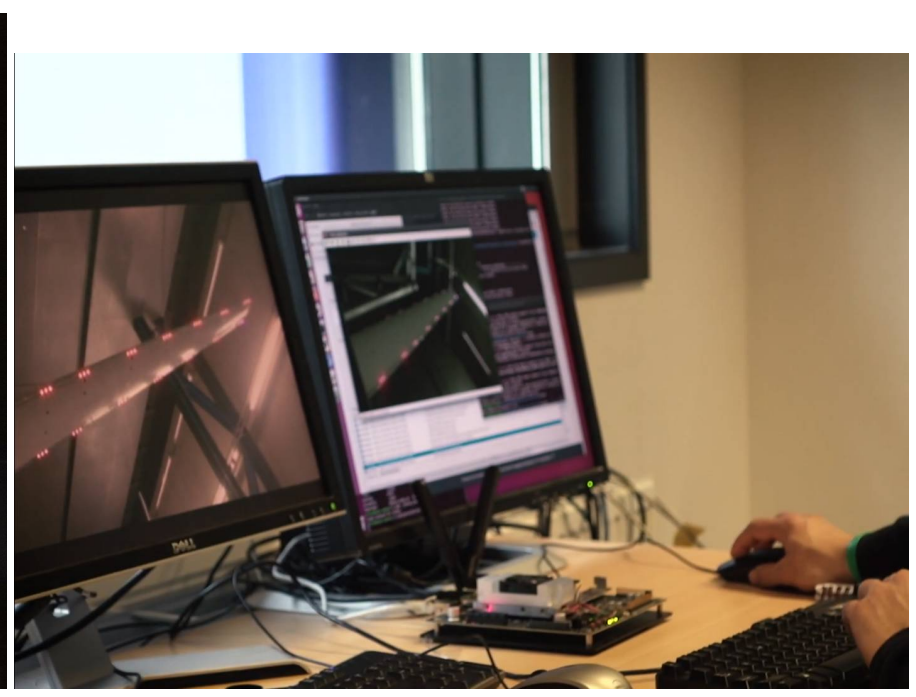

(b) Data collection and live tracking with a Jetson TX2 [15].

Figure 9 Experimental Setup 


\section{Results and Discussion}

\section{A. Measured wing response}

In Fig. 10 the responses to 1-cos gust and sweep input signals are shown. The time history signals correspond to the measurements taken at the location of marker ID 1. The labelling and notation of the marker IDs for the vibrometer measurement system is shown in Fig. 9a. Figures 10a and10b show the response of the wing to a single gust input and a sweep signal, respectively. The solid curves correspond to measurement by the laser vibrometer sampled at $400 \mathrm{~Hz}$; the dotted line is a spline model of this response sampled at the capture intervals by the leading edge camera. This spline model is required to obtain synchronised measurement points between the laser vibrometer data and the image sequences for comparison. The camera images were collected at approximately $40 \mathrm{~Hz}$, with the Nyquist frequency well above the expected resonance frequency of the wing of $\approx 5 \mathrm{~Hz}$.

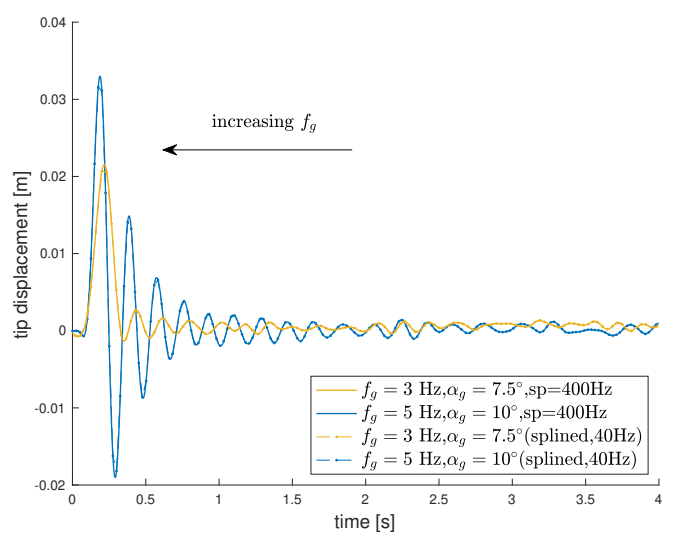

(a) 1-cos gust input signal for varying gust vane angles and gust frequencies at flow velocity of $V=30 \mathrm{~m} / \mathbf{s}$.

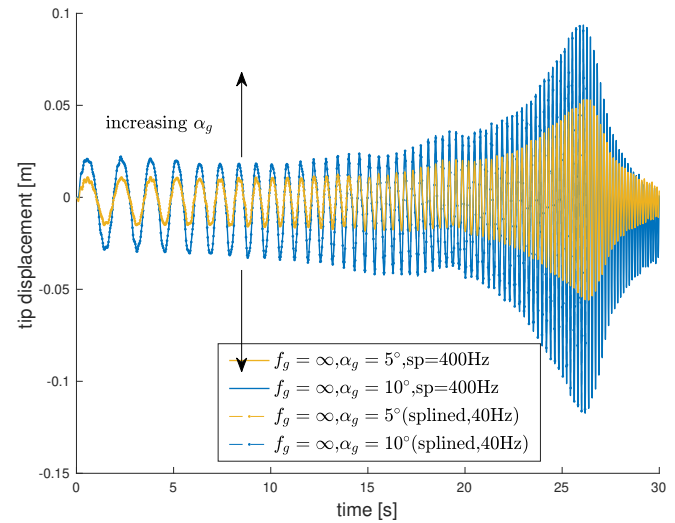

(b) Sweep input signal with varying gust vane angles at flow velocity of $V=30 \mathrm{~m} / \mathrm{s}$.

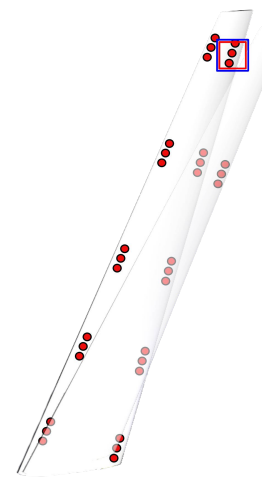

(c) Tracker location.

Figure 10 The laser vibrometer measurement (solid line), of the tip displacement of marker ID 1, sampled and splined at capture intervals of the leading edge at $\approx 40 \mathrm{~Hz}$ (dotted line).

The deflection of the wing at static motion conditions (various free stream velocities) is shown in Fig. 11b shows the baseline static deflections obtained at varying free stream velocities. In Fig. 11a the dynamic response of the wing as a result of the gust excitation is shown in a spatial and time-domain representation.

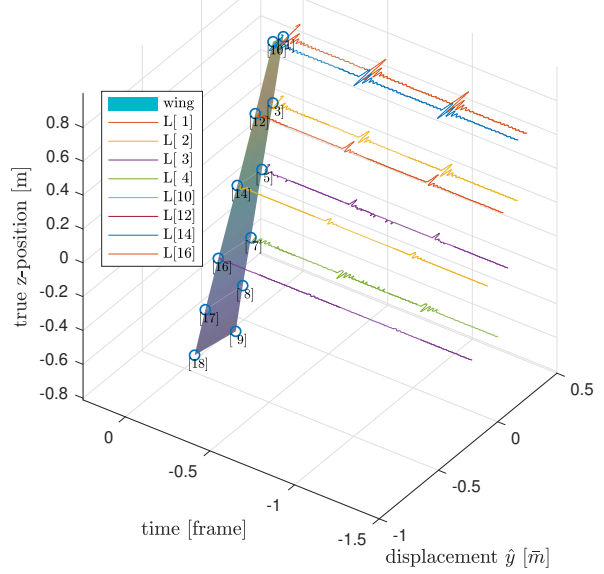

(a) 3D view and marker tracking in time domain.

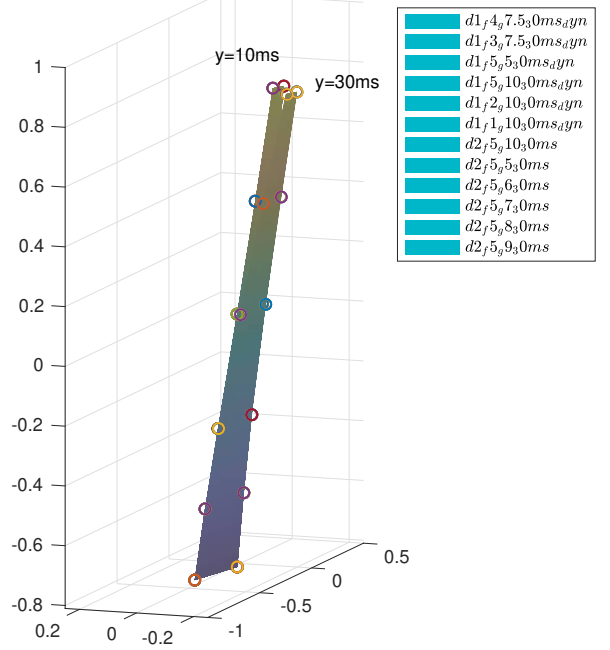

(b) Baseline static deflections at varying $V$.

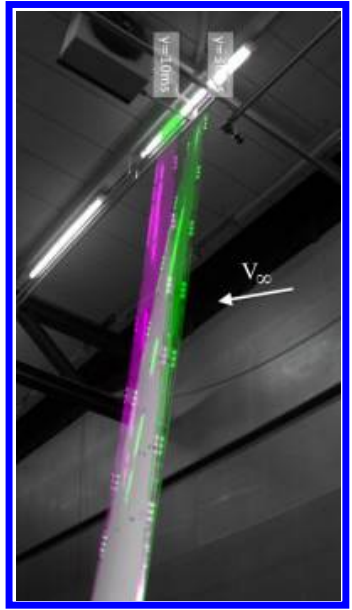

(c) Wind direction.

Figure 11 Spatial and time representation of the series of wing deflection under static and dynamic loads. 


\section{B. Gust parameter sensitivity and wing response analysis}

To better understand the requirements and the needed performance of the visual tracking, the wing response to various gust inputs was studied. Here, the sensitivity of wingtip response (marker ID1) to 1-cosine gust input $\left(\alpha_{g}, f_{g}, V\right)$ parameters was measured.

Observing Fig. 10a the effect of the change of input gust frequency is seen by comparing the high frequency (blue) to low frequency (yellow). At higher frequencies, above $3 \mathrm{~Hz}$, the wing response resembles more an impulse response, while at lower frequencies, it resembles more a 1-cosine input signal. Fig. 12a shows the full range of frequencies (1-5 $\mathrm{Hz}$ ). This shift in response type appears to happen between the $3 \mathrm{~Hz}$ (yellow) and $4 \mathrm{~Hz}$ (red) lines. This can be explained as for narrower 1-cosine gust inputs, the response tends to attain the characteristics of an impulse, and the energy is absorbed in a narrower time span by the wing. On contrast below the $3 \mathrm{~Hz}$, the response is spread out over a longer time. The purple line in Fig. 12b shows this larger spread between $0-0.5$ seconds. This observation indicates that the higher frequency responses are more critical for visual tracking in particular above $3 \mathrm{~Hz}$. Therefore for the performance of 5 $\mathrm{Hz}$ gust are at the centre of the following discussions.

Further observations can be made regarding the sensitivity of the gust vane angle and the free stream velocity. Fig. $12 \mathrm{~b}$ shows the responses for varying gust vane angles of the gust generator ranging from 5 to 10 degrees (blue to cyan lines), while the free stream velocity is kept at $30 \mathrm{~ms}$. Higher gust vane angles generate larger amplitude cosine waves corresponding to larger input energy and higher lift. As seen from Fig. 12b this results in larger amplitudes of the wing response. Similarly, larger free stream velocities result in higher amplitudes. Fig. 12c shows how the response amplitude is increased by over 6 fold, from approximately 0.005 to 0.032 meters, between $10 \mathrm{~ms}$ (red) and $30 \mathrm{~ms}$ (blue). Both of these motion responses are relevant for visual tracking, as higher amplitudes in shorter time span challenge the capture and processing frame rate of the visual tracking, and lower amplitudes, the sensitivity threshold for pixel activity, directly depending from the image resolution. However, it must be noted that larger amplitudes are more relevant for controller feedback. The differences between the gust vane angles are impacting on the response amplitude, therefore, mainly the gust vane angle of 10 degrees is discussed.

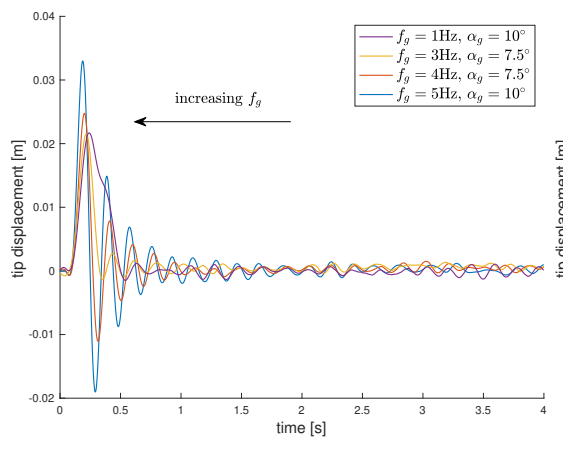

(a) Varying frequency $f_{g}$ at constant $\mathrm{V}$ of 30 (b) ms.

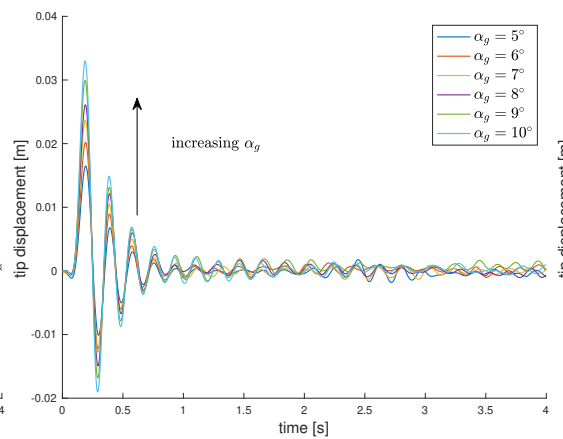

Varying gust vane angle
of $30 \mathrm{~ms}$ and $f_{g}$ of $5 \mathrm{~Hz}$

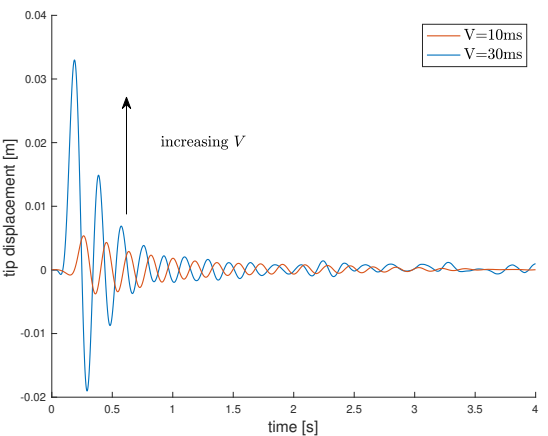

(c) Varying free stream velocity $V$ at constant $\alpha_{g}$ of $10{ }^{\circ}$ and $f_{g} \mathbf{5 H z}$.

Figure 12 Effect of gust input parameters on the measured wing response.

Regarding the sweep input, it is observed that the increasing gust vane angle results in a larger amplitude. This shown by the wider amplitude band of the 10-degree signal (blue) versus 5-degree signal (yellow). The lower amplitude signal (yellow) is expected to be more challenging for visual tracking as less activity is expected in the subsequent image frames.

\section{Motion reconstruction and analysis in Frequency and time-domain}

To quantify the measured wing response and the tracked wing motion from the image sequences, comparisons were made in terms of displacements and the frequency content in Fig. 13. The laser vibrometer measured response (in meters) was reconstructed with the KCF-EKF (green) pipeline 6. To obtain the reconstructed displacements, the camera setup was calibrated and the corresponding KCF-EKF point pairs from two image streams triangulated with the DLT method. The details of the triangulation approach are given in $[9,20]$. The obtained 3D coordinates were then transformed to align the laser vibrometer measurement. Similar to laser vibrometer measurement, the baseline static displacement was subtracted to obtain the transient displacement of $d y$ as indicated by the last block in the diagram 6 . 
Observing the reconstructed displacement of the tip marker in Fig. 13a, a good agreement with the laser measurements is found in terms of phase of the response. The reconstruction seems to overshoot the response at the amplitude peaks. In particular, after the first peak, the difference between the laser and reconstruction is approximately $3.5 \mathrm{~mm}$. In the first peak, the difference is significantly lower, below $2 \mathrm{~mm}$. This difference can be attributed for three reasons, namely, (i) the splining of the laser reference measurement tends to undershoot the peak due to lower sampling rate, (ii) transformation between laser measurement and absolute reconstructed coordinates and (iii) stereo calibration errors. The latter factor plays a significant role in the quality of the tracking and requires to carefully calibrate the entire volume of the 3D space where the motion takes place. In the current case, the calibration could only be performed when the wing was already installed, which prevented the cover of the spatial domain of the markers. Furthermore, errors in measurement between the orientation and location of the camera setup to the reference system, the laser vibrometer, also play a role in the comparison. Additionally, a fourth reason is the smoothening of the peaks by the Kalman-filtering and the lag introduced. However, these can be more easily adjusted by tuning the Kalman filter parameters with the KCF-AEKF approach to rely more on the KCF tracker measurement. It must be noted that this, however, would also reduce the robustness of the tracking to occlusions hence a trade-off must be made.

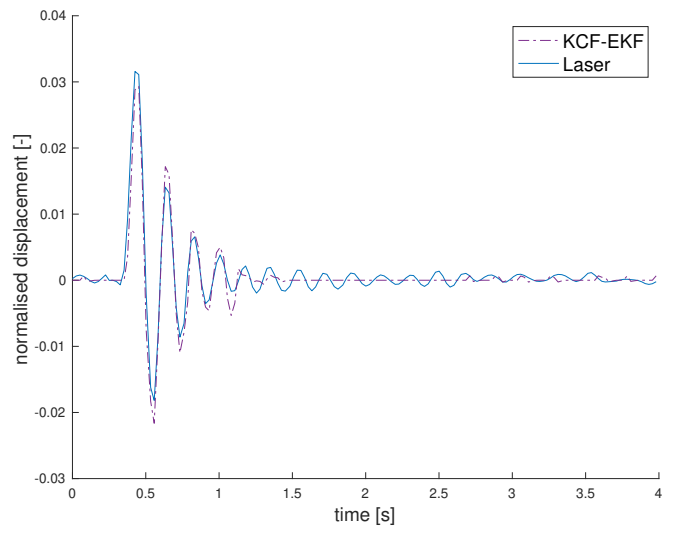

(a) Comparison response of marker ID 1.

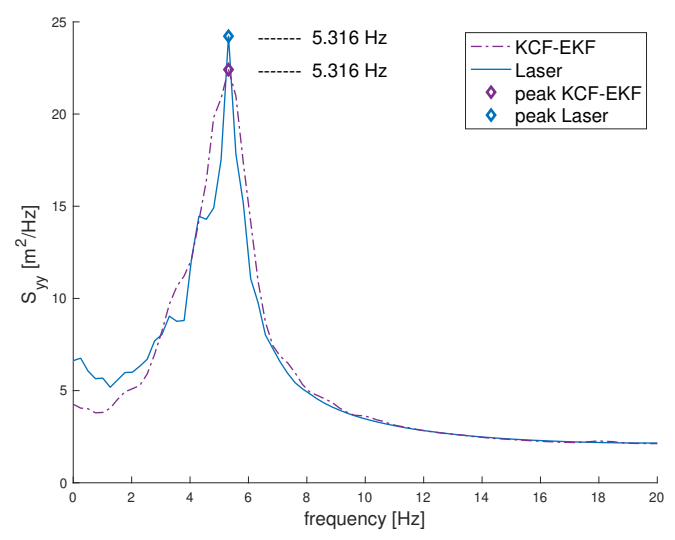

(b) Auto-PSD of the response signal at marker ID 1.

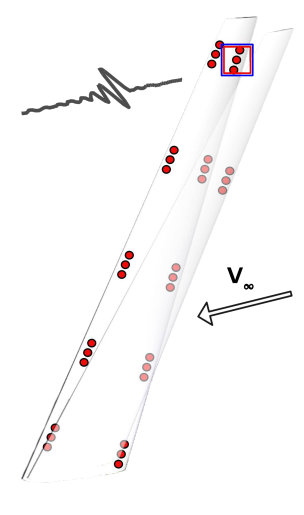

(c) Tracker location.

Figure 13 Comparison of the response and the frequency spectrum for laser vibrometer versus image tracking pipelines with DBSCAN and the Disjoint-set data structure for run R1 and marker ID 3.

Alternatively, the good agreement of the phase of the reconstructed motion can be further analysed employing a frequency domain analysis. Here, the measurements were compared in terms of the power spectral densities (PSD) of the measured output. The main objective of this analysis was to understand whether the image tracking methods could correctly identify the frequency content of the measured signal compared to the reference measurement provided by the laser vibrometer. In this context, the aim was not to extract the system's frequency response function, hence no cross-correlation was performed of the input to the output signal, and the power spectrum of the output alone provided sufficient ground for comparison. The auto-PSD of the output signal, $S_{y y}$, was calculated according to the following definition:

$$
S_{y y}(\omega)=\int_{-\infty}^{\infty} R_{y y}(\tau) e^{-j \omega \tau} d \tau
$$

where the integral in the expression is the Fourier transform of the auto-correlation function $R_{y y}$ of the output signal (marker displacement). Figure 13b shows the frequency content of the image sequence corresponding to the responses of marker ID 1. As can be observed from Fig. 13b, the tracking method can provide a good estimate of the first resonant frequency of the wing. The result shows that both peaks of the spectral densities align at $5.316 \mathrm{~Hz}$, and despite underestimation of the amplitude peaks, the resonance region is captured well with the reconstructed signal. Overall observing the time and frequency domain analysis results suggest that the oscillatory motion of the wing can be well captured and reconstructed with the relatively low-resolution cameras (1.3 megapixels) at capturing rate of $40 \mathrm{~Hz}$. The accuracy can be further improved by higher resolution cameras and higher capture rate. 


\section{Conclusion and Recommendation}

In this study, a method was proposed for robust visual tracking and reconstruction of flexible wing motion controller state feedback. The method consisted of a tracker pair composed of a purely visual filter, a high-speed Kernelized Correlation Filter (KCF) [6], paired with an Augmented Extended Kalman Filter (AEKF), allowing to adaptive estimate the states and parameters of the system exhibiting oscillatory motion. The method consisting of KCF-EKF, without Kalman filter augmentation, was validated experimentally on a real-time image stream of a very flexible wing subjected to gust excitation in the OJF (Open Jet Facility) at the Delft University of Technology. From the measured experimental data obtained with the laser vibrometer system, the effect of gust frequency, the gust vane angle and the free stream velocity were studied on the wing response, to demonstrate the requirements of the visual tracking and reconstruction. The reconstructed motion of the wingtip markers from KCF-EKF tracking showed good agreement with the reference measurement provided by the vibrometer. Frequency and time-domain analysis and comparison of the data showed that the resonance peak could be captured well with the reconstructed response from the visual tracking. The results suggested that the oscillatory motion of the wing can be well captured and reconstructed with the relatively low-resolution cameras (1.3 megapixels) and a non-invasive sensing system. The low resolution allows us reaching higher capture rates for better accuracy of the reconstruction. A further study is planned to analyse the performance of the Extended and Augmented Kalman filter for varying motion conditions under the presence of uncertainty in the visual information in the form of marker failure.

\section{Acknowledgments}

The authors would like to thank Johannes Dillinger of the German Aerospace Center (DLR) for providing the wing model and assistance during the experimental data collection. Furthermore, the authors would like to thank the colleagues of the Aerospace Structures and Materials department for helping to assemble the gust generator.

\section{References}

[1] Burner, A. W., and Liu, T., "Videogrammetric model deformation measurement technique," Journal of Aircraft, Vol. 38, No. 4, 2001, pp. 745-754. https://doi.org/10.2514/2.2826.

-[2] Corke, P. I., "Visual Control of Robot Manipulators - a Review," Visual Servoing: Real-Time Control of Robot Manipulators Based on Visual Sensory Feedback, World Scientific, 1993, pp. 1-31. https://doi.org/10.1142/9789814503709_0001.

-[3] Wang, X., "Intelligent multi-camera video surveillance: A review," Pattern recognition letters, Vol. 34, No. 1, 2013 , pp. 3-19.

-[4] Belbachir, A. N., Smart cameras, Vol. 2, Springer, 2010. https://doi.org/10.1007/978-1-4419-0953-4.

- [5] Mkhoyan, T., Thakrar, N. R., De Breuker, R., and Sodja, J., "Design of a Smart Morphing Wing Using Integrated and Distributed Trailing Edge Camber Morphing," ASME 2020 Conference on Smart Materials, Adaptive Structures and Intelligent Systems, American Society of Mechanical Engineers, 2020, pp. -. https://doi.org/10.1115/smasis2020-2370, URL https://asmedigitalcollection.asme.org/SMASIS/proceedings/SMASIS2020/84027/Virtual,Online/1090388.

-[6] Henriques, J. F., Caseiro, R., Martins, P., and Batista, J., "High-Speed Tracking with Kernelized Correlation Filters," IEEE Transactions on Pattern Analysis and Machine Intelligence, Vol. 37, No. 3, 2015, pp. 583-596. https://doi.org/10.1109/TPAMI. 2014.2345390, URL http://ieeexplore.ieee.org/document/6870486/.

[7] Gray, R. M., “Toeplitz and circulant matrices: A review,”, 2006. https://doi.org/10.1561/0100000006.

[8] Mkhoyan, T., “tmkhoyan/adaptiveClusteringTracker: Initial public release,”, 2019. https://doi.org/10.5281/zenodo.3561015, URL https://zenodo.org/badge/latestdoi/224665601.

- [9] Mkhoyan, T., de Visser, C. C., and De Breuker, R., "Parallel Real-Time Tracking and 3D Reconstruction with TBB for Intelligent Control and Smart Sensing Framework," AIAA Scitech 2020 Forum, American Institute of Aeronautics and Astronautics (AIAA), 2020, p. 2252. https://doi.org/10.2514/6.2020-2252.

[10] Mkhoyan, T., de Visser, C. C., and De Breuker, R., "Adaptive Real-Time Clustering Method for Dynamic Visual Tracking of Very Flexible Wings," AIAA Scitech 2020 Forum, American Institute of Aeronautics and Astronautics (AIAA), 2020, p. 2250. https://doi.org/10.2514/6.2020-2250.

[11] Jongkind, K., Falkmann, A., and van der Veer, H., “Open Jet Facility,” , 2020. URL https://www.tudelft.nl/lr/organisatie/ afdelingen/aerodynamics-wind-energy-flight-performance-and-propulsion/facilities/low-speed-wind-tunnels/open-jetfacility/. 
[12] Lancelot, P., Sodja, J., and De Breuker, R., "Investigation of the unsteady flow over a wing under gust excitation," 17th International Forum on Aeroelasticity and Structural Dynamics, IFASD 2017, Vol. 2017-June, 2017. URL https://repository. tudelft.nl/islandora/object/uuid \{\%\}3A2caf1e73-3de2-4067-803d-8a52c9050fea?collection=research.

[13] Polytec, “Polytec SINGLE-POINT VIBROMETERS,”, 2020. URL https://www.polytec.com/us/vibrometry/products/\{ \protect\ T1 textdollar\} single-point-vibrometers/.

[14] Ritter, M., Meddaikar, Y. M., and Dillinger, J. K., "Static and dynamic aeroelastic validation of a flexible forward swept composite wing," 58th AIAA/ASCE/AHS/ASC Structures, Structural Dynamics, and Materials Conference, 2017, 2017 , p. 0637. https://doi.org/10.2514/6.2017-0637, URL https://arc.aiaa.org/doi/pdf/10.2514/6.2017-0637.

[15] Mkhoyan, T., “tmkhoyan/cvyamlParser: Initial public release,”, nov 2019. https://doi.org/10.5281/zenodo.2703498, URL https://zenodo.org/badge/latestdoi/184505001.

[16] Basler AG, "Basler ace acA1300-30gm - Area Scan Camera," , 2019. URL https://www.baslerweb.com/en/products/cameras/ area-scan-cameras/ace/aca1300-30gm/.

[17] Computar, “Computar Machine Vision Lens Catalog,” Tech. rep., Computar, 2017. URL https://computar.com/resources/ files $\left\{_{-}\right\}$v2/1551/Computar\{_\}FAv0817.pdf.

[18] "Embedded Computer Vision Real Time? Nvidia Jetson TX2 + VisionWorks toolkit - Myzhar's MyzharBot and more...", 2020. URL https://www.myzhar.com/blog/embedded-computer-vision-real-time-nvidia-jetson-tx2-visionworks-toolkit/.

[19] Bradski, G., "The OpenCV Library,” Dr Dobbs Journal of Software Tools, Vol. 25, 2000, pp. 120-125. https://doi.org/10.1111/ 0023-8333.50.s1.10, URL http://opencv.willowgarage.com.

[20] Mkhoyan, T., “tmkhoyan/parallelTrackingTBB: initial release,”, dec 2019. https://doi.org/10.5281/zenodo.3564905, URL https://doi.org/10.5281/zenodo.3564905. 